University of Warwick institutional repository: http://go.warwick.ac.uk/wrap

This paper is made available online in accordance with publisher policies.

Please scroll down to view the document itself. Please refer to the repository record for this item and our policy information available from the repository home page for further information.

To see the final version of this paper please visit the publisher's website. Access to the published version may require a subscription.

Author(s): Kandala N-B, Ji C, Cappuccio FP, Stones W R Article Title: The Epidemiology of HIV infection in Zambia Year of publication: 2008 Link to published version: http://www.informaworld.com/smpp/content $\sim$ content=a793825133 $\mathrm{db}=\mathrm{all}$ $\sim$ tab=content $\sim$ order $=$ pubdate 


\title{
The Epidemiology of HIV infection in Zambia
}

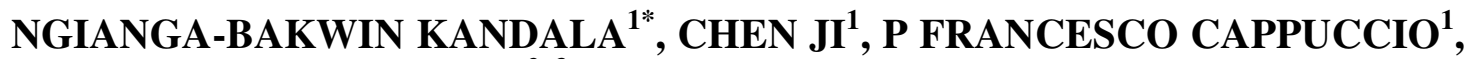 AND R WILLIAM STONES ${ }^{2,3}$}

${ }^{1}$ Clinical Sciences Research Institute, Warwick Medical School, Clifford Road Bridge, CV2 2LU Coventry, UK.

${ }^{2}$ Director, Centre for AIDS Research, University of Southampton, Southampton SO17 1JB

${ }^{3}$ Chair of Obstetrics and Gynaecology, Aga Khan University Hospital, Nairobi, P.O. Box 30270-00100 GPO, Nairobi.

\begin{abstract}
Population surveys of health and fertility are an important source of information about demographic trends and their likely impact on the HIV/AIDS epidemic. In contrast to groups sampled at health facilities they can provide nationally and regionally representative estimates of a range of variables. Data on HIV sero-status were collected in the 2001-2 Zambia Demographic and Health Survey (ZDHS) and made available in a separate data file in which HIV status was linked to a very limited set of demographic variables. We utilized this data set to examine associations between HIV prevalence, gender, age and geographical location.
\end{abstract}

We apply the generalized geo-additive semi-parametric model as an alternative to the common linear model, in the context of analyzing the prevalence of HIV infection. This model enables us to account for spatial auto-correlation, non-linear, location effects on the prevalence of HIV infection at the disaggregated provincial level ( 9 provinces) and assess temporal and geographical variation in the prevalence of HIV infection, while simultaneously controlling for important risk factors.

$54 \%$ of the overall sample of 3950 was female. The overall HIV positivity rate was 565 (14.3\%). The mean age at HIV diagnosis for male was 30.3 (SD: 11.2) and 27.7 (SD: 9.3) for female respectively.

Lusaka and Copperbelt have the first and second highest prevalence of AIDS/HIV (marginal odds ratios of 3.24 and 2.88 respectively) but when the younger age of the urban population and the spatial auto-correlation was taken into account Lusaka and Copper belt were no longer among the areas with the highest prevalence. Nonlinear effects of age at HIV diagnosis were also discussed and the importance of spatial residual effects and control of confounders on the prevalence of HIV infection. 
The study was conducted to assess the spatial pattern and the effect of confounding risk factors on AIDS/HIV prevalence and to develop a means of adjusting estimates of AIDS/HIV prevalence on the important risk factors.

Controlling for important risk factors such as geographical location (spatial auto-correlation), age structure of the population, gender gave estimates of prevalence that are statistically robust. Researchers should be encouraged to use all available information in the data to account for important risk factors when reporting AIDS/HIV prevalence. Where this is not possible, correction factors should be applied, particularly where estimates of AIDS/HIV prevalence are pooled in systematic reviews. Our maps can be used for policy planning and management of AIDS/HIV in Zambia.

\section{INTRODUCTION}

Population surveys of health and fertility are an important source of information about demographic trends and their likely impact on the HIV/AIDS epidemic. In contrast to groups sampled at health facilities they can provide nationally and regionally representative estimates of a range of variables. The Demographic and Health Surveys (www.measuredhs.com) are a well established source of reliable population level data with a substantial focus on knowledge of HIV/AIDS and sexual behaviour. However, linking individual survey records with the results of HIV testing has been considered undesirable owing to the absolute need to maintain confidentiality on this most sensitive of health topics. Data on HIV sero-status were collected in the 2001-2 Zambia Demographic and Health Survey (ZDHS) and made available in a separate data file in which HIV status was linked to a very limited set of demographic variables.

\section{Background: Characteristics of the study area}

Zambia is an African country at the heart of sub-equatorial Africa with a population of about 12 million and a population growth rate of about 1.66 percent/year [Global 
Health Facts, 2007]. With a gross national product (GNP) of 380 US\$ /person/year, around two-thirds of the population lives on less than a dollar a day ,and Zambia is among the 20 least economically developed countries in the world [1].

However, Zambia is very rich in mineral deposits. This was the backbone of its economy until the 1970s, when its economy was severely affected by a fall in global copper prices.

More than a quarter (40\%) of its population live in two urban areas: Lusaka (capital city where $10 \%$ of the population lives) and in the industrial towns of the Copperbelt. The rest of population of Zambia is very sparse, particularly the west and the northeast, and farming is the main activity for the majority of people. HIV prevalence is considerably higher in these urban areas (54\% of all adults living with HIV or AIDS ) and the highest HIV prevalence has been recorded among pregnant women in Lusaka, Kabwe, Ndola, Mongu, and the cross-border tourist and trading centres: Chipata and Livingstone [2].

Zambia has the fourth highest adult prevalence in the world and the social and economic impact of the pandemic is overwhelming. According to UNAIDS/WHO estimates in 2005 [3], $17 \%$ of people aged 15-49 years old were living with HIV or AIDS and 57\% of them were women. The rate of infection among young women aged 15-19 were six times compared to males of the same age [4]. In 2005, an estimated 100,000 people died of AIDS and 710,000 children were left orphans by AIDS. Life expectancy, as a result of this high mortality has fallen below 37 years [3]. HIV/AIDS has also set back the rates of economic growth in Zambia, although, more recently the country's economic prospects have been transformed by debt relief and 
rising copper prices but it remains to be seen whether these gains will be converted into human development.

Mobility of population in Zambia is common place and the risk of infection is higher among these people and among those whom they contact. People often work as seasonal labourers or migrant fishermen/women, truck drivers and they spend long periods away from their regular partners, thus spreading the virus from one area to another.

Despite economic difficulties Zambia has invested in health. Responses to HIV and AIDS in Zambia have aimed to prevent HIV transmission; to care for people who are infected and affected; and to reduce the personal, social and economic impact of AIDS. Since 2002, the government of Zambia has been engaged in a challeging antiretroviral (ARV) treatment programme and progress on the reduction of HIV prevalence is encouraging although, HIV prevalence remains at a very high level [5]. Scaling-up the provision of treatment and reducing the number of new infections to so many millions of people is a challenge for the government of Zambia to alliviate the burden of this pandemic.

\section{MATERIAL AND METHODS}

\section{Data Source}

The objectives, organisation, sample design, and questionnaires used in the 2001-2 DHS survey have been described in detail elsewhere [6]. It was a nationally representative household survey of adult men and women (15-49 years). All samples were stratified, the 18 strata formed by the urban and rural areas of each province. The questionnaire included information on socio-economic indicators, fertility patterns, health and care practices, health knowledge, anthropometric status, and information 
about sexual behaviour and knowledge of HIV/AIDS prevention. The survey was carried out by the Central Statistical Office with technical assistance from Macro International, funded by US Agency for International Development (USAID).

\section{Methods}

The methods of the ZDHS survey are described, together with an overview report (7). Briefly, this was a random probability sample of women age 15-49 and men age 1559. Approximately 2,500 men and a similar number of women were asked to give blood for syphilis and HIV testing. Samples for HIV testing were dried blood spots on a filter paper card taken from a venous blood specimen. A three stage testing procedure was used with $10 \%$ of the negative samples retested and discordant results tested by Western Blot. 2073 women and 1877 men were tested for HIV representing $79 \%$ and $73 \%$ of those identified as eligible for testing in the survey. The sample was weighted to account for the unbalance population size of $68.2 \%$ from rural areas and $31.8 \%$ from urban areas.

We utilized this data set to examine associations between HIV status, gender, age and area of residence so as to improve the precision of the estimates for better public health policy.

Figure 1 shows the geographic distribution of marginal odds ratios from the data and Figure 2 shows marginal probabilities of HIV as a function of age for both males and females. Preliminary results indicated an age by gender interaction $(\mathrm{p}<0.001)$. Noting the non-linear association of age and odds of HIV infection we examined the nonlinear association of the effect of age on the posterior odds ratio of HIV infection for men and women separately in Figure 3. Figure 4 shows the total residual spatial 
effects are shown in Figure 4 after accounting for spatial auto-correlation in the data arising from the population mobility (migration) and other important risk factors in Zambia.

\section{Model Description}

Instead of using region-specific dummy variables to capture the geographical dimension and account for spatial auto-correlation on HIV infection in Zambia, we applied a novel approach by exploring spatial patterns of the prevalence of HIV infection and possible nonlinear effects within a simultaneous, coherent regression framework using a geo-additive semi-parametric mixed model. The model employed a fully Bayesian approach developed in Fahrmeir and Lang [8] using Markov Chain Monte Carlo (MCMC) techniques for inference and model checking.

The model used for this investigation has been described elsewhere ${ }^{1}[8-9-10]$. The response variable in this application is defined as $y_{i}=1$ if HIV sero-positive and $y_{i}=0$ otherwise. The standard measure of effects is the posterior odds ratio (OR).

The traditional methods of the study of HIV/AIDS prevalence astonishingly neglect the geographical location, spatial auto-correlation and nonlinear effects of covariates, which is likely to result in misleading conclusions in prevalence of diseases. Additionally the more important impact of this neglect would be an underestimation

\footnotetext{
${ }^{1}$ Briefly, we replace the strictly linear predictor $\eta_{i}=x^{\prime} \beta+w_{i}^{\prime} \gamma \quad, \quad$ (1) with a logit link function with dynamic and spatial effects $\operatorname{Pr}\left(y_{i}=l \mid \eta_{t}\right)=\mathrm{e}^{\eta_{t}} /\left(1+\mathrm{e}^{\eta t}\right)$ with a geo-additive semi-parametric predictor $\mu_{\mathrm{i}}=\mathrm{h}\left(\eta_{t}\right): \eta_{l}$ $=f_{l}\left(x_{i l}\right)+\ldots+f_{p}\left(x_{i p}\right)+f_{\text {spat }}\left(s_{i}\right)+w^{\prime}{ }_{i} \gamma(2)$

where $h$ is a known response function with a logit link function and $f_{l}, \ldots, f_{p}$ are non-linear smooth effects of the metrical covariates (age at HIV diagnosis) and $f_{\text {spat }}\left(s_{i}\right)$ is the effect of the spatial covariate $s_{i} \in\{1, \ldots, S\}$ labelling the region in Zambia. Covariates in $w_{i}^{\prime}$ are usual categorical variables such as gender and urban-rural residence. Regression models with predictors as in (2) are sometimes referred to as geo-additive models. P-spline priors are assigned to the functions $f_{1}, \ldots, f_{p}$ and for $f_{\text {spat }}\left(s_{i}\right)$ we used Markov random field prior [ 8-9-10]. The analysis was carried out using BayesX version 0.9, software for Bayesian inference based on Markov Chain Monte Carlo simulation techniques.
} 
of standard errors of the fixed effects that inflates the apparent significance of the estimates [9-10]. Our analysis included this correlation structure and account for the dependence of neighbouring community (mobile population i.e. truck drivers, seasonal labourers or migrant fishermen/women, spending long periods away from their regular partners) in the model. Since the ZDHS data are based on a random sample within provinces. That is, the structured component introduced here allows us to 'borrow strength' from neighbours in order to cope with the sample variation of the province effect and obtain estimates for areas that may have inadequate sample sizes or be un-sampled. This gives more reliable estimates of the fixed effect standard error.

A failure to take into account the posterior uncertainty in the spatial location (province) would overestimate the precision of the prediction of the prevalence of HIV infection in regions with inadequate sample size.

In the descriptive analysis, Table 1 was generated to show the prevalence of HIV infection by age, gender and regions. Chi-square tests and Mann-Whitney tests were used to investigate the association between factors and disease. Multivariate analysis shows the significance of the posterior odd ratio estimates of fixed effects (Table 2), non-linear effects (Figure 3) and residual spatial effects (Figure 4).

\section{RESULTS}

$54 \%$ of the overall sample of 3950 was female. The overall HIV sero-prevalence was $565(14.3 \%)$. The mean age at survey diagnosis of HIV sero-positivity for men was 30.3 (SD: 11.2) and 27.7 (SD: 9.3) for women respectively. It should be noted that as part of the sample age selection strategy, men were sampled from age 15 up to older 
ages of 59 and women were sampled from age 15 to 49. As expected from the sampling strategy, the mean age of men at the time of sero-diagnosis of HIV was older (30.3 SD: 11.2) compared to women.

Table 1: Prevalence of HIV/AIDS infection by baseline characteristics: ZDHS 20012).

\begin{tabular}{|l|l|l|l|}
\hline Variables & Negative: N (\%) & Positive: N (\%) & P-value \\
\hline Number & $3,384(85.7)$ & $565(14.3)$ & \\
\hline Gender & & & \\
\hline Male & $1,605(88.3)$ & $212(11.7)$ & \\
\hline Female & $1,779(83.4)$ & $353(16.6)$ & $<0.001$ \\
\hline Place of residence & & & \\
\hline Urban & $966(76.9)$ & $290(23.0)$ & \\
\hline Rural & $2,418(89.8)$ & $275(10.2)$ & $<0.001$ \\
\hline Age at diagnosis & & & \\
\hline Age1 (<24 years) & $1,503(92.5)$ & $122(7.5)$ & \\
\hline Age2 (25 \& 35 years) & $1,014(78.8)$ & $273(21.2)$ & \\
\hline Age3 (36 \&49 years) & $740(82.3)$ & $159(17.7)$ & \\
\hline Age4 (50 \& 59 years) & $127(92.0)$ & $11(8.0)$ & $<0.001$ \\
\hline Region of residence & & & \\
\hline Central & $478(84.9)$ & $85(15.1)$ & \\
\hline Copperbelt & $400(80.2)$ & $99(19.8)$ & \\
\hline Eastern & $336(86.4)$ & $53(13.6)$ & \\
\hline Luapula & $296(88.9)$ & $37(11.1)$ & \\
\hline Lusaka & $335(77.9)$ & $95(22.1)$ & \\
\hline Northern & $568(92.1)$ & $49(7.9)$ & \\
\hline North-western & $402(90.7)$ & $41(9.3)$ & \\
\hline Southern & $329(82.7)$ & $69(17.3)$ & \\
\hline Western & $240(86.6)$ & $37(13.4)$ & \\
\hline
\end{tabular}

Table 1 compares the prevalence of HIV infection, demographic and geographical characteristics at baseline in the 565 cases with HIV and in the 3,384 sero-negative subjects at the time of the survey. The prevalence of HIV infection was higher among women compared with men $(16.6 \%$ vs. $11.7 ; \mathrm{p}<0.001)$, higher in urban areas compared with rural areas $(23.0 \%$ vs. $10.2 \%$; $<<0.001)$, higher in the age groups ranging from $25-35$ years and 36-49 years but lower in the younger $(<25$ years) and 
older (50-59 years) age groups $(21.2 \%, 17.7 \%$ vs. $7.5,8.0 \% ; \mathrm{p}<0.001)$, very high in Lusaka (22.1\%) and Copper belt (19.8\%) provinces followed by Southern (17.3\%) and Central (15.1\%) provinces, but lower in the Northern (7.9\%) and North-western (9.3\%) provinces compared with Eastern (13.6\%), Luapula (11.1\%) and Western $(13.4 \%)$ provinces.

[FIGURE 1 ABOUT HERE]

The spatial variation of the observed HIV prevalence in Zambia is drawn in Figure 1 by plotting the marginal odds ratio of HIV at regional level. Northern region is set as reference. All the regions are classified into 6 marginal odds ratio bands in which North Western region shares the same band and colour with Northern region. The map also shows that the highest three bands of marginal odds ratio are in the central and southern part of Zambia. Further, Lusaka and Copperbelt, as the most developed areas, have the highest marginal odds ratios (3.24 and 2.88 respectively).

[FIGURE 2 ABOUT HERE]

We calculated observed probability of HIV infection in the survey and plot it against age of all respondents. Figure 2 shows there is a bell shaped non-linear relationship between age and probability of HIV infection. A watershed is present between 30-34 years at which people have the highest observed probability in the survey for both men and women. Before 30, the probability of HIV infection rises quickly as age increases. This implies that age has an almost linear relationship with the probability 
of HIV infection. People who are over 34 years are likely to have a declining risk of HIV infection although the variation of the probability increases rapidly at the same time as age continues to increase. The figure also shows there is no HIV positive case detected in the survey for men who are 50, 52, 53, 57 and 58 years old, but this is likely to represent an artefact of the small numbers sampled at the older ages.

Table 2: Posterior odds ratio of the fixed effect parameters for the risk of HIV infection (Model 2).

\begin{tabular}{|l|l|l|l|l|}
\hline Variables & Odds ratio & Std. Error & 95\%CI & P-value \\
\hline Gender & & & & \\
\hline Female & 1.59 & 0.09 & $1.32,1.89$ & $<0.001$ \\
\hline Male & 1 & reference & & \\
\hline Place of residence & & & & \\
\hline Urban & 2.73 & 0.10 & $2.13,3.28$ & $<0.001$ \\
\hline Rural & 1 & Reference & & \\
\hline
\end{tabular}

Table 2 shows the results of the posterior odds ratio of the fixed effects covariates included in Model 2. Gender and place of residence are the factors considered in the model. The nonlinear effects of age and the total residual spatial effects of the region are shown in Figure 3 and Figure 4 respectively. Male respondents and rural area were set as reference groups in the respective factors. Women and men have significantly different risks of getting infected with HIV. Compared to men, women were much more likely to be HIV sero-positive (odds ratio=1.59, 95\% CI= $[1.32,1.89]$ ). A higher risk of HIV infection was found among people from urban areas $(2.73[2.13,3.28])$ compared to those in rural areas. These results are consistent with the univariate results in Table 1.

In the observed marginal data we saw Lusaka and Copperbelt showing a high odds ratio (Figure 1). After controlling for the spatial auto-correlation, age-gender profile and its urban status, we found that the prevalence of HIV infection in Lusaka and 
Copperbelt are no longer as high as might be expected. Similarly, Southern region showed a much higher risk of HIV infection once we controlled for the spatial autocorrelation, age, and gender and residence status (Figure 4).

Figure 4 left indicated that people living in Southern province had the highest risk of HIV infection than those in Northern Province. Red represents a higher risk of HIV infection while green denotes a lower risk. The risk of HIV infection in the two most developed regions: Lusaka and Copperbelt appear to be much less than observed. The risk of HIV infection in Luapuala and North Western provinces are the lowest in the country. The right panel of Figure 4 shows the posterior probability maps of the risk of HIV infect at a $80 \%$ credible interval. The provinces in black indicated a significant positive spatial effect, while the provinces in white implied a significant negative spatial effect. The rest of the provinces (in grey) had no significant effect on HIV infection. Their corresponding probability maps (Figure 4 right) suggested that there is no significant difference of the risk HIV infection in these provinces compared to Northern province.

\section{[FIGURE 3 ABOUT HERE]}

The nonlinear effects of age at HIV diagnosis are shown in Figure 3 revealed that both genders have rapidly increasing risk of HIV infection before age 30 and almost linearly declining risk y afterwards (Figure 3). Women appeared to have higher risk of being infected with HIV than men in all age groups. The 20-24 age groups showed the largest gap of the risk of HIV infection between male and female.

[FIGURE 4 ABOUT HERE] 
In sum, the flexible modelling of the region-specific effects paints a much more nuanced picture than was presented by the observed marginal odds ratio and thus give a better impression of the spatial variation of HIV infection. Moreover, the geoadditive semi-parametric Bayesian approach used is able to identify subtle influences of the age at HIV diagnosis on the risk of HIV infection.

\section{DISCUSSION}

HIV/AIDS is one of the greatest health, social and economic problems in Zambia country and nearly one million people are HIV positive or have AIDS. An estimated 100,000 died of the epidemic in 2004 (3). UNAIDS/WHO estimated at the end of 2005 that $17 \%$ of people aged 15-49 years old were living with HIV or AIDS. 57\% of this population with HIV or AIDS, according to the same source were women (4).

In this study we identified the spatial pattern of the risk of HIV infection in Zambia. We found that the risk of HIV infection remains high in central and southern part of the country in both estimated and observed map of HIV prevalence.

Many socio-economic factors may account for the above finding. The development of this landlocked country relies much on the train service which is offered mainly in the central area covering Lusaka, Copperbelt and Central regions. Among these regions, Lusaka and Copperbelt are the most developed and urbanised. However, the economy of Zambia as a whole has been deteriorating since the drop of copper prices in 1970s. Furthermore, although rural poverty apparently negatively affects local public health and makes people face economic hardship (1), an urbanised area with high population density faces a more severe burden of HIV infection. In the 2001-2 ZDHS, the 
prevalence of HIV infection was $23.0 \%$ in urban areas compared to $10.2 \%$ in rural area (Table1). As shown in Table 2, people in urban areas have almost three fold increase (OR: $2.73 ; 95 \%$ CI: 2.13-3.23) risk of HIV infection compared to those in rural areas.

In addition, the migration of people from countries sharing a border with Zambia may affect the current HIV/AIDS prevalence in the country. Prior to the 1990s, there was a high prevalence of HIV in the Democratic Republic of the Congo and Uganda which lie to the north of Zambia. Later on, the high prevalence moved to the eastern and southern part of Africa. UNAIDS (11) reports that Botswana and Zimbabwe are among the countries in which HIV prevalence exceeds $30 \%$. The high odds ratios of the risk of HIV infection in regions like Southern and Lusaka can be explained partially in this perspective.

The migration of specific groups of people to urban areas, such as seasonal labourers and commercial sex workers, is considered to play an important role in the spread of HIV/AIDS (12). Seasonal labourers who work away from home are likely to keep short term relationships with women or sometimes have sex with sex workers, which contribute to the pattern of spread of HIV/AIDS across the country. Furthermore, Agyei-Mensah's study (13) discovered from the case of Ghana that commercial sex workers were likely to migrate from less developed areas to those with much improved economic conditions. A study (14) showed that two thirds of sex workers in Zambia were HIV positive and they may risk loss of income if they request condom use with clients to avoid the spread of HIV inside the country. 
Our most important finding is illustrated in the contrast between Figures 1 and 4 . A pronounced change of the odd ratios in Lusaka and Copperbelt is observed following application of the model described here. The spatial distributions of the risk of HIV infection we showed are of course influenced by the variable selection in the model. Place of residence and spatial distribution are both chosen as the factors in geoadditive regression model. However, it is possible that the place of residence shared the effect of geographic distribution of the regions, especially in Lusaka and Copperbelt as the country is highly urbanised and almost one-half of the country's twelve million people are concentrated in a few urban zones.

Rapid increase in sero-prevalence in both genders before the age of 30 is shown in Figure 3. People in Zambia generally report sexual debut at an early age; some studies $(7,15,17)$ revealed that the average age of debut is around 17 in females and 17.5 in males. For men, the rapid age related increase of the risk of HIV infection probably may be fuelled by their greater tendency to move from rural to urban areas to look for employment. As young people, they are more sexually active and might have more opportunities to be exposed to unprotected sex than others. For women, many of them have their first sex with men on average 5 years older than them. This may explain why at the same age young women have a higher risk of HIV infection than young men.

Another factor contributing to the age related discrepancy between male and female risk of HIV infection is gender inequity. Women have much less access to education and generally are economically dependent on men. Even marriage does not bring many sexual health benefits to women because of a higher frequency of sex, low rates 
of condom use and their husbands' risky sexual behaviour (16). While an effective way to prevent STIs and HIV infection, condom use is mainly dominated by men and it is difficult for women to insist on the use of a condom. Thus current HIV programme focuses on other interventions such as the female condom and vaginal microbicides for HIV prevention. The latter may have great potential as a 'woman initiated' method, should current clinical trials provide proof of efficacy. Other negative gender pressures on Zambian women include poverty in widowhood, because they do not have the right to inherit the property of the family (11). Another problem is that other household members may also have sex with these women, following a tradition termed sexual cleansing to dispel evil. This tradition substantially increases women's risk of HIV infection.

\section{CONCLUSION}

We identified the spatial pattern of the risk of HIV/AIDS infection in Zambia at the regional level. A higher than expected prevalence is revealed in the central and southern parts of the country after controlling for spatial auto-correlation and other important risk factors. Various socio-economic factors have impacts on the current situation of HIV/AIDS epidemic in Zambia. Although sharing the same pattern of age effect on the risk of HIV/AIDS infection, men and women have a different probability of infection mainly due to gender inequality and lifestyle. Poverty still largely hampers the development of health care interventions. However, more efforts are being made by the government and many international organisations, and recent achievements have been reported in many studies and mass media. Geographic understanding of the pattern of this epidemic using models as presented here can be helpful for the government to target health care intervention plans more effectively. 


\section{REFERENCES}

1. Rural Poverty Portal, (2006). Rural poverty in Zambia. Available from [http://www.ruralpovertyportal.org/english/regions/africa/zmb/index.htm] (accessed Sept 18 Nov 2007).

2. UNAIDS/WHO Epidemiological Fact Sheet - 2004 Update, Zambia

3. UNAIDS/WHO 2006 Report on the global AIDS epidemic

4. UNAIDS/WHO 2006 Report on the global AIDS epidemic

5. Zambia Summary Country Profile for HIV/AIDS Treatment Scale-up, WHO/UNAIDS, July 2004

6. Central Statistical Office Republic of Zambia, Central Board of Health Republic of Zambia, Macro O. Zambia Demographic and Health Survey 2001-2. Calverton, Maryland. USA: Central Statistical Office, Central Board of Health and ORC Macro, 2003

7. Central Statistical Office , (2004). [http://www.cpc.unc.edu]. Zambia Sexual Behaviour Survey 2003. 2004; Available from [http://www.cpc.unc.edu/measure/publications/index.php] (accessed 16 Nov 2006).

8. Fahrmeir L, Lang S., 2001. Bayesian Inference for Generalized Additive Mixed Models Based on Markov Random Field Priors. Applied Statistics (JRSS C). 50: 201220.

9. Kandala N-B: Bayesian Geo-additive modelling of Childhood morbidity in Malawi. Applied Stochastic Models in Business and Industry, 2006; 22:139-154.

10. Kandala N-B, C Ji , N Stallard , S Stranges \& FP Cappuccio Spatial Analysis of Risk Factors for Childhood Morbidity in Nigeria. American Journal of Tropical Medicine \& Hygiene, 2007 (In press).

11. UNAIDS/WHO (2002). AIDS Epidemic Update: 2002. Available from [http://www.who.int/hiv/pub/epidemiology/epi2002/en] (accessed 16 Nov 2006).

12. Mayer, JD., (2005). The geographical understanding of HIV/AIDS in sub-SaharanAfrica. Norwegian Journal of Geography ; 59: 6-13.

13. Agyei-Mensah, S., (2001). Twelve years of HIV/AIDS Interventions in Sub-SaharanAfrica: Contrasting Conceptualizations of 'Risk' and 'Spaces' of Vulnerability. PHD dissertation, University of Washington.

14. AVERT (2006). Introduction to AIDS in Zambia. Available from [http://www.avert.org/aids-zambia.htm] (accessed 16 Nov 2006) 
15. Zaba, B., Pisani, E., Slaymaker, E., and Ties Boerma, J., (2004). Age at first sex: understanding recent trends in African demographic surveys. Sex Transm Infect ; 80: 28-35. 16. First global analysis of sexual behaviour, (2006). Available from: [http://www.lshtm.ac.uk/news/2006/sexualbehaviour.html\#one] (accessed 16 Nov 2006). 17. Garcia-Calleja, JM., Gouws, E., Ghys, PD., (2006). National population based HIV prevalence surveys in sub-Saharan Africa: results and implications for HIV and AIDS estimates. Sex Transm Infect ; 82(Suppl III): iii64-70. 
Figure 1: Map of marginal odds ratios of HIV prevalence in Zambia (DHS 2001)

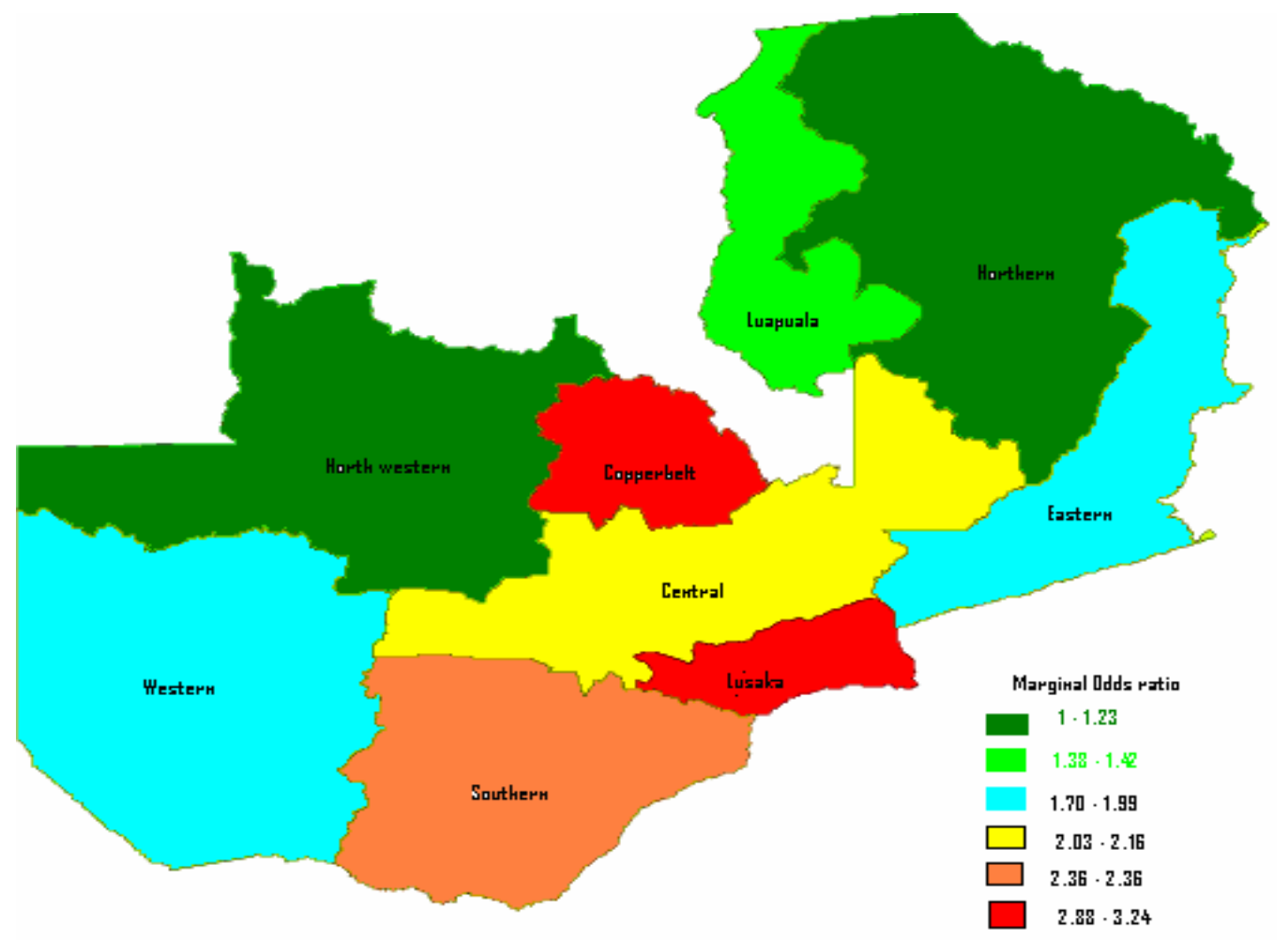


Figure 2: Marginal probability of HIV positive status as a function of age (ZDHS, 2001-2)

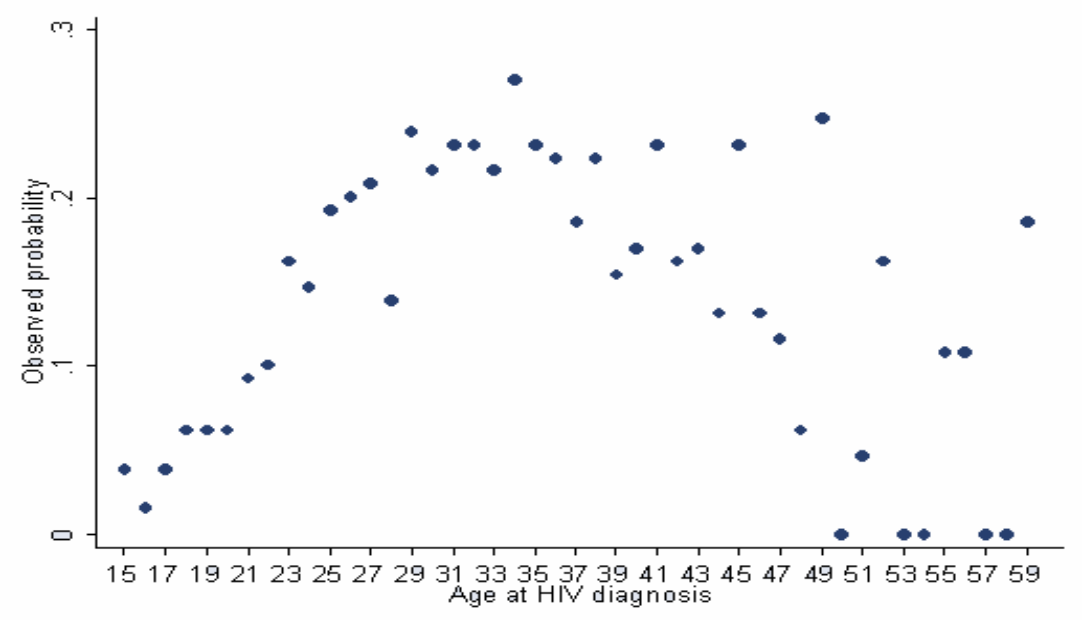

Figure 3: Gender specific estimated nonlinear effects of age at HIV/AIDS diagnosis.

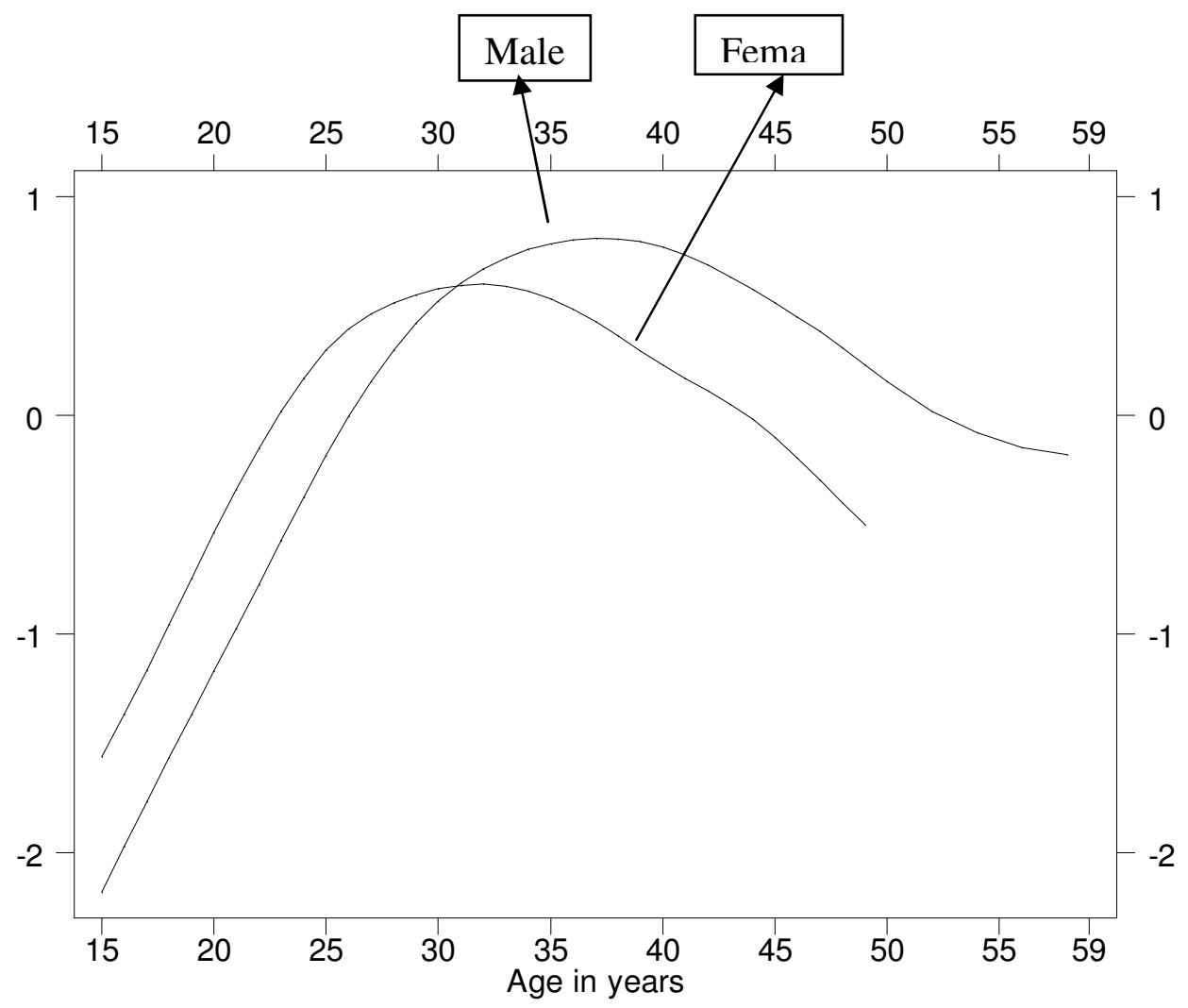


Figure 4: Total residual spatial provincial effects (left) and $80 \%$ posterior probability map (right) of the risk of HIV infection in Zambia.

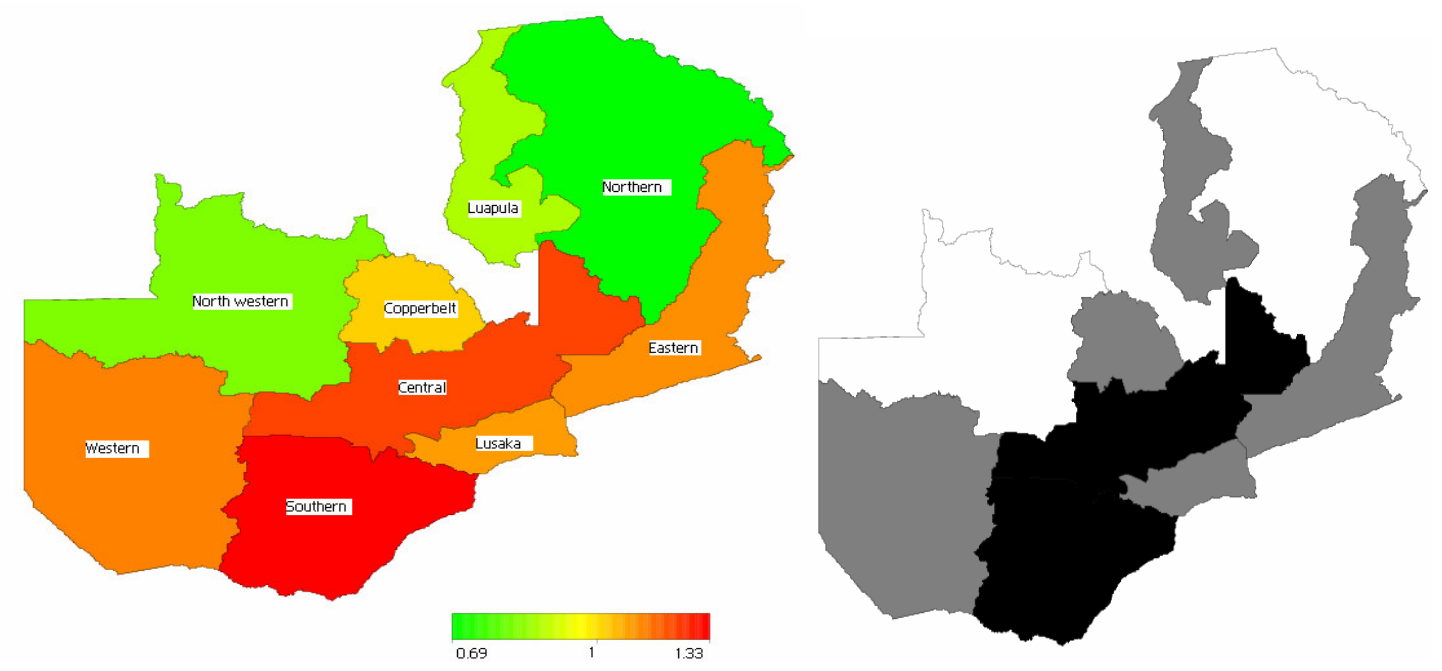

Red coloured - high risk

Black coloured - significant positive spatial effect

Green coloured - low risk

White coloured- significant negative spatial effect Grey coloured - no significant effect 